\title{
Aplicações da Matriz Insumo-Produto em análises ambientais no Brasil: uma revisão sistemática de literatura
}

\author{
Raul Asseff Castelao' \\ Celso Correia de Souza" \\ Daniel Massen Frainer ${ }^{\prime \prime \prime}$
}

\section{Resumo}

Diversos fatores têm contribuído para o aumento da degradação ambiental do planeta. Entre eles, o aumento da população e, consequentemente, aumento da demanda por produtos e serviços, sendo considerado um dos principais fatores geradores de externalidades para o meio ambiente. De forma a identificar ferramentas para minimizar os níveis de degradação do meio ambiente, diversas iniciativas têm sido desenvolvidas de modo a descrever a relação da economia com o meio ambiente. Como sendo um destes instrumentos, a matriz insumo-produto possibilita a descrição da relação de interdependência dos setores de uma economia com o meio ambiente de forma mais específica. Neste sentido, o objetivo deste trabalho foi o de apresentar uma revisão sistemática de literatura (RSL) sobre o uso de matriz insumo-produto com enfoque a questões ambientais no Brasil. Para tanto, foi feito uma RSL em periódicos internacionais e nacionais de modo a: quantificar e analisar os estudos disponíveis que tratam da relação do meio ambiente e matriz insumo. Foi possível identificar a existência de 1.667 artigos que trabalham com a técnica de matriz insumo-produto e o meio ambiente, sendo que deste total, 19 estudos abordam essa temática para o Brasil entre os anos de 2000 a 2018.

Palavras-chave: Meio ambiente; Produção; Matriz insumo-produto

\section{Abstract}

Several factors have contributed to the increasing environmental degradation of the planet. Among them, the increase of the population and, consequently, increase of the demand for products and services, being considered the main factors generating externalities for the environment. In order to identify the tools to minimize the levels of degradation of the environment, the strategy of saving energy with the environment. As an instrument, an inputoutput matrix enables a more specific description of the interdependence relationship of the sectors of an economy to the environment. This article is a cross-reference of the literature review in RSL. The data was not found in the financial data and national studies in the data and method in the analysis of the data of methods. It was possible to identify the existence of 1,667 articles that work with the input-output technique and the environment. Of this total, 19 studies approached this theme for Brazil between the years 2000 and 2018.

Keywords: Environmental; Productions; Input-output matrix 


\section{Introdução}

Tendo em vista o aumento da população e, por consequência, o aumento dos níveis de produção, torna-se primordial compreender os elos e repercussões entre o setor econômico produtivo e o meio ambiente. A preocupação em procurar entender os impactos das atividades econômicas e da sociedade sobre o meio ambiente tem ganhado mais espaço nas agendas de pesquisas e tomadores de decisão. O número de debates e trabalhos que procuram descrever as relações entre economia e o meio ambiente evidenciam a crescente preocupação em busca de análises nessa ótica (SCHMITZ e BITTENCOURT, 2017).

Conforme as sociedades se tornam mais ricas, há uma exigência cada vez maior de demanda por materiais e energia para sustentar as suas atividades e o seu padrão de vida. Entre os anos de 1900 a 2005, a extração total de materiais de biomassa vegetal e animal, minérios e minerais industriais, minerais de construção e combustíveis fósseis aumentou oito vezes no mundo todo (KRAUSMANN et al., 2009). A partir do aumento populacional e, principalmente, de pessoas morando e trabalhando nos centros urbanos, os sistemas de produção e de prestação de serviços públicos passarão a demandar 180 bilhões de toneladas de biomassa até 2050, quase três vezes a demanda atual (UNEP, 2016).

A redução do rendimento de determinadas culturas em algumas áreas do planeta e a crescente taxa de extinção de algumas espécies animais e vegetais em função das alterações climáticas estão causando perdas irreversíveis (SULSTON et al., 2013). O cenário atual é o da degradação ambiental pela intensa exploração e a consequente diminuição da oferta de recursos naturais, derivado do crescimento populacional e industrial no mundo, com consequente aumento do consumo (BROWM, 2011; ROYAL SOCIETY, 2012).

Com a publicação do trabalho de Dennis Meadows, Donella Meadows e Jorden Randers, intitulado Limits to growth, a interface entre o meio ambiente, a economia e a sociedade ganharam destaque em função de que o trabalho descreve uma relação conflituosa e com sérias repercussões sobre a sociedade, por não se levar em consideração o meio ambiente como uma variável no processo produtivo de geração de riqueza das nações. Desde então, inúmeros tem sido os esforços na busca em equalizar da melhor maneira a relação entre a economia, meio ambiente e a sociedade (MEADOWS et al., 1972).

Neste sentido e, tendo como objetivo central demonstrar que a relação entre economia e meio ambiente deveria ser levada em consideração nos moldes de produção e consumo, Nicholas Georgescu-Roegen produziu o seminal The Entropy Law and the Economic Process in Retrospect em 1971, revelando que o fluxo produtivo econômico da época possuía incoerências, sendo a principal delas, o fato de que o modelo não levava em consideração questões como, por exemplo, da oferta de recursos naturais e como estes se relacionavam mediante suas transformações físicas (lei da entropia) (CECHIN e VEIGA, 2010).
Desta forma, não seria mais possível apresentar o diagrama do fluxo circular de produção fechado sem a variável do meio ambiente, mas sim, com a necessária consideração da oferta de recursos naturais aonde, a partir deste novo fluxo de produção e consumo, tem-se uma nova forma de produção a partir do modelo clássico, ou seja, para cada nível de produção tem-se a geração de externalidades sobre o meio ambiente e a própria sociedade, uma vez que a preocupação básica é o crescimento econômico (CECHIN e VEIGA, 2010).

A partir do debate que surgiu nos anos de 1970 em função do trabalho de Meadows e Jorden e de Nicholas Georgescu-Roegen, cunhou-se a teoria do decrescimento, a qual se baseia na hipótese de que o crescimento econômico não é sustentável para o ecossistema global (LOUETTE, 2007). Precisa-se de um novo modelo econômico para o século XXI, um modelo em sincronia com a terra e seus sistemas de suporte naturais (BROWN, 2011).

De forma a sistematizar o debate sobre a relação do meio ambiente com a economia e a sociedade, a ONU realizou em 1972 a primeira conferência sobre o meio ambiente, em Estocolmo. Um dos resultados desta iniciativa foi à criação do Programa das Nações Unidas para o Meio Ambiente (PNUMA) que em 1986, por meio da Comissão Mundial sobre o Meio Ambiente e Desenvolvimento (CMMD), cunhou o conceito de desenvolvimento sustentável, como sendo: "aquele que atende às necessidades das gerações atuais sem comprometer a capacidade das gerações futuras de atenderem a suas necessidades e aspirações” (CMMD, 1987).

O desenvolvimento sustentável só faz sentido e só é possível para a sociedade apenas se entendido como desenvolvimento sem crescimento, ou seja, com melhorias qualitativas de vida levando em consideração a capacidade de regeneração e assimilativas dos recursos naturais (DALY, 2004). O conceito de desenvolvimento sustentável pode ser entendido também como aquele que promove condições ambientais que resulta da geração de bens e serviços a serem absorvidos pela geração atual sem comprometer as condições ambientais que garantiria igual, ou melhor, padrão de vida para as gerações futuras (FEIJÓ et al., 2011).

Neste sentido, o desenvolvimento sustentável é entendido como um compromisso com o avanço do bem estar humano, com a compreensão de que este desenvolvimento deve ocorrer dentro dos limites ecológicos da bioesfera (MORAN et al., 2008).

Contudo, para que a sociedade alcance o desenvolvimento sustentável é necessário um monitoramento e análise do fluxo produtivo relacionado com o comportamento humano via demanda de produtos e serviços, bem como, a oferta de recursos naturais. Este monitoramento pode atender diversos enfoques, podendo ser um compromisso da humanidade com o meio ambiente ou ser um compromisso de caráter social e econômico, procurando atingir determinado objetivo como, por exemplo, a geração de empregos que protejam o meio ambiente (HARDI, 1997).

Tomando como base o fluxo produtivo de Nicholas 
Georgescu-Roegen (1970), compreende-se ser necessário o uso da técnica de matriz insumo-produto como uma das formas de mensurar e analisar a relação entre a economia, à sociedade e o meio ambiente. A matriz insumo-produto (MIP) é o instrumento com base na análise microeconômica e da contabilidade social que permite conhecer os fluxos de bens e serviços produzidos nos setores de determinada economia, destinados a servir de insumos a outros setores para atender a demanda das famílias (CARVALHEIRO, 1998).

A base conceitual do modelo de matriz insumo-produto deriva da abordagem de François Quesnay (1758), a Tableu de Economique. Neste trabalho, Quesnay desenvolve um sistema que descreve as inter-relações entre os setores produtivos a época (LEONTIEF, 1936). Wassily Leontief em 1936 publica o seminal trabalho Quantitative Input and Output Relations in the Economic Systems of the United States no periódico The Review of Economics and Statistics tendo como base o trabalho de Quesnay, sendo esta publicação a primeira experiência prática da matriz insumo-produto, e foi desenvolvida para descrever a inter-relação e o fluxo produtivo da economia norte americana (RICHARDSON, 1978).

A matriz de insumo-produto é uma estrutura de quadros que revela, a partir da desagregação dos dados de produção de determinada economia, os elos de dependência e interdependência dos setores produtivos (ROSSETTI, 1995). A matriz insumo-produto pode ser caracterizada, portanto, como uma fotografia da economia de determinado país ou região, demonstrando quais os setores que suprem os outros setores de serviços e produtos e, quais são supridos por quem (GUILHOTO, 2011).

Utiliza-se a matriz insumo-produto para mensurar esta relação, pois, devido ao fato de que o desenvolvimento sustentável deve partir da premissa do nível de produção, e como se dá esse fluxo, e não da teoria da utilidade econômica, para descrever como a economia possui interfaces com o meio ambiente (DALY, 2002).

As tabelas de matriz insumo-produto vêm sendo utilizadas como instrumento que possibilita descrever a relação entre a economia e o meio ambiente, possibilitando a identificação e estudo dos problemas ambientais derivados do fluxo produtivo (MIRANDA, 1980). O modelo de matriz insumo-produto permite que seja possível descrever a origem e destino da produção de cada setor e medir quais os impactos exógenos de mudanças na economia sobre as variáveis endógenas, inclusive, sobre o meio ambiente (FEIJÓ et al., 2011).

Em função do aumento da preocupação da mensuração dos reflexos da ação antrópica sobre o meio ambiente, assim como, a busca de uma compreensão mais aplicada deste processo de interação, este trabalho teve como objetivo desenvolver uma revisão de literatura sobre o uso do modelo de matriz insumo-produto, levando-se em consideração o meio ambiente no Brasil.

O objetivo geral deste estudo é o de demonstrar que o uso da técnica de matriz insumo-produto, com seus diversos modelos, têm contribuído para a construção de análises mais descritivas do reflexo de variações nos níveis de produção e consumo sobre o meio ambiente, servindo, portanto, de um instrumental mais adequado para mensuração da relação entre a sociedade, a economia e o meio ambiente podendo, inclusive, subsidiar a formulação de políticas públicas com vistas à preservação e manutenção do meio ambiente.

O objetivo específico é, em primeiro lugar, quantificar o número de trabalhos que utilizam a MIP como método para analisar a relação da economia com o meio ambiente; segundo, apresentar as principais técnicas de matriz insumo-produto; descrever a aplicação dos modelos de matriz insumo-produto no Brasil e quais os principais resultados dessas aplicações e, por fim; como a técnica de MIP tem sido aperfeiçoada com o propósito de descrever de forma mais analítica a inter-relação entre a economia e o meio ambiente.

\section{Material e métodos}

A abordagem da pesquisa é de caráter indutivo e qualitativa cujo procedimento de pesquisa é a revisão sistemática de literatura (RSL). Do ponto de vista dos objetivos do presente estudo, utilizou-se da técnica exploratória para descrever a interface entre o uso da matriz insumo-produto em assuntos relacionados ao meio ambiente.

A RSL é considerada método que permite identificar, avaliar e interpretar toda a pesquisa disponível relevante para determinado tema de pesquisa (KITCHENHAM e CHARTERS, 2007). Com o uso da RSL, é possível apresentar novos resultados sobre temas específicos por meio de análise e sistematização de informações publicadas sobre o mesmo (DENYER e TRANFIELD, 2009).

A RSL torna-se útil, pois possui a capacidade de ajudar o pesquisador no dimensionamento e na compreensão a cerca do estado da arte de determinado assunto ou área de pesquisa, possibilitando identificar pesquisas já realizadas, lacunas e até mesmo o que falta pesquisar sobre o assunto (CONFORTO et al., 2011).

De forma a sistematizar a pesquisa dos trabalhos, foi utilizada a lei de Zipf, terceira lei clássica da bibliometria. A lei de Zipf procura descrever a relação entre palavras em um trabalho e a contagem das palavras no mesmo. A formulação desta lei parte do princípio de que, ao listar as palavras usadas em um trabalho de maneira decrescente de frequência, a posição de determinada palavra multiplicada pela sua frequência é igual a uma constante (ARAÚJO, 2006). A equação da lei de Zipf é dada pela equação 1 .

$$
r \times f=\mathrm{k}
$$

Onde $\mathrm{r}$ é a posição da palavra, f é a sua frequência e $\mathrm{k}$ é a constante. Sendo assim, Zipf formulou o princípio do menor esforço, havendo uma economia do uso de palavras, aonde a tendência de que as palavras mais usadas incidam o assunto do trabalho (ARAÚJO, 2006).

A pesquisa bibliográfica foi realizada em sete etapas divididas em duas fases (quadro 2). 
Quadro 2 - Etapas da pesquisa de RSL

\begin{tabular}{|l|l|l|}
\hline Fase & Etapa & Descrição \\
\hline \multirow{4}{*}{1} & Etapa 1 & Definição das palavras-chaves \\
\cline { 2 - 3 } & Etapa 2 & Definição dos periódicos \\
\cline { 2 - 3 } & Etapa 3 & Análise de conteúdo/título \\
\cline { 2 - 3 } & \multirow{2}{*}{ Etapa 4 } & $\begin{array}{l}\text { Análise estatística descritiva dos dados } \\
\text { encontrados }\end{array}$ \\
\hline \multirow{3}{*}{2} & Etapa 5 & $\begin{array}{l}\text { Recorte para estudos relacionados ao } \\
\text { Brasil }\end{array}$ \\
\cline { 2 - 3 } & Etapa 6 & Análise de conteúdo \\
\cline { 2 - 3 } & Etapa 7 & Conclusões \\
\hline \multirow{2}{*}{ Fonte: Adaptado de KITCHENHAM e CHARTERS, 2007. }
\end{tabular}

A primeira etapa consistiu na definição das palavras-chaves utilizadas para pesquisar os descritores: matriz insumo-produto, meio ambiente e matriz insumo-produto hibrida, considerando o idioma português e inglês. Os critérios de recorte de periódicos (etapa 2) foram dois: impacto do periódico, ou seja, com classificação de qualidade acima de B1 e escopo alinhado com o tema matriz insumo-produto e o meio ambiente. Nesta etapa, o levantamento foi feito nas bases de pesquisas eletrônicas de periódicos e artigos, Scielo, JsTor, Ecology and society e Elsevier, com o objetivo de identificar os trabalhos que foram publicados tendo em seu escopo conceitos de matriz insumo-produto ligada ao meio ambiente no Brasil, que é objetivo do trabalho. A pesquisa foi realizada no período de fevereiro a abril de 2018.

$\mathrm{Na}$ etapa 3, foram feitas leituras de títulos e resumo dos artigos de modo a identificar a exata relação do estudo com a questão da MIP e o meio ambiente. Após essa etapa, foi possível fazer a análise estatística descritiva dos resultados (etapa 4). A etapa 5 foi feito recorte para trabalhos levando em consideração o Brasil e, após esse filtro, análise dos conteúdos (etapa 6) e a conclusão (etapa 7).

A fase 1 consiste na abordagem geral, considerando trabalhos feitos em diversos locais. A fase 2 trata-se dos trabalhos que abordam o Brasil.

Os dados recolhidos foram organizados usando o Microsoft Excel ${ }^{\circledR}$ para a geração dos resultados da estatística descritiva.

A razão de se fazer uma RSL da matriz insumo-produto para análise da relação entre economia e meio ambiente, é descrever os modelos precursores no uso deste método na década de 1960 e 1970, bem como suas principais características e como esta técnica tem sido utilizada para estudos no Brasil.

Como o método da matriz insumo-produto teve seu início de forma mais robusto no final da década de 1930, o período temporal da pesquisa bibliográfica inicia na primeira publicação de Wassily Leontief (1936), a respeito do uso da matriz insumo-produto, ainda sem o enfoque para o meio ambiente, e finaliza com as publicações até maio de 2018.

\section{Resultados e discussão}

Levando-se em consideração a crescente importância da problemática ambiental, tem havido esforços no sentido de incorporar a dimensão ambiental nos modelos de desenvolvimento das nações, e o uso do modelo de insumo-produto tem sido empregado para esta finalidade. Neste sentido, diversos estudos usando a matriz insumo-produto e a relação com o meio ambiente, principalmente, para descrever os impactos de poluição ambiental, foram desenvolvidos na década de 1960 (MILLER e BLAIR, 2009).

Os modelos de matriz insumo-produto que possuem como objetivo descrever a relação meio ambiente e economia pode ser classificado em três categorias básicas (MILLER e BLAIR, 2009):

I. Modelos Generalizados de Entrada-Saída - estes são formados pelo aumento de linhas e/ou colunas e seus respectivos coeficientes para refletirem a geração de poluição em detrimento do nível de produção;

II. Modelos Econômicos-Ecológicos - esses modelos resultam do aumento da estrutura da matriz para incluir setores ecossistêmicos, onde os fluxos entre os setores econômicos e do ecossistema se dão de acordo com um modelo inter-regional insumo-produto e;

III. Modelos de Produtos por Setor - esses modelos expressam os impactos ambientais a partir de uma matriz de relação produto versus setor.

Esses modelos apresentados são considerados a base em se tratando da aplicação da matriz insumo-produto em questões relacionadas ao meio ambiente como, por exemplo, os impactos de poluição em detrimento do aumento do nível de produção em determinada economia.

Mesmo o uso da matriz insumo-produto no Brasil, para fins de mensurar a relação de interdependência da atividade econômica e seus efeitos para com o meio ambiente, tenha sido iniciado nos anos de 1970, esta técnica passa a ter maior número de publicações a partir da década de 2000. A hipótese para este destaque temporal consiste no aperfeiçoamento da construção da matriz insumo-produto, de melhores recursos tecnológicos e disponibilidade de dados para a regionalização da matriz insumo-produto permitindo, desta forma, a ampliação do uso desta técnica ao longo do tempo.

Ao analisar a evolução das publicações, é possível notar que na década de 2000 a um número maior de trabalhos desenvolvidos com esta temática e, especificamente, a partir de 2013, este número aumenta consideravelmente (Figura 2).

Embora os trabalhos de análise de relação da economia e o do meio ambiente, com base no modelo da matriz insumo-produto, terem sido desenvolvidos no final da década de 1960 em outros países, no Brasil o uso desta abordagem teve seu início na década de 1980 . No caso brasileiro, as primeiras matrizes de insumo-produto da economia nacional foram desenvolvidas pelo Instituto Brasileiro de Geografia e Estatística (IBGE) no final da 
Figura 2 - Publicações por ano

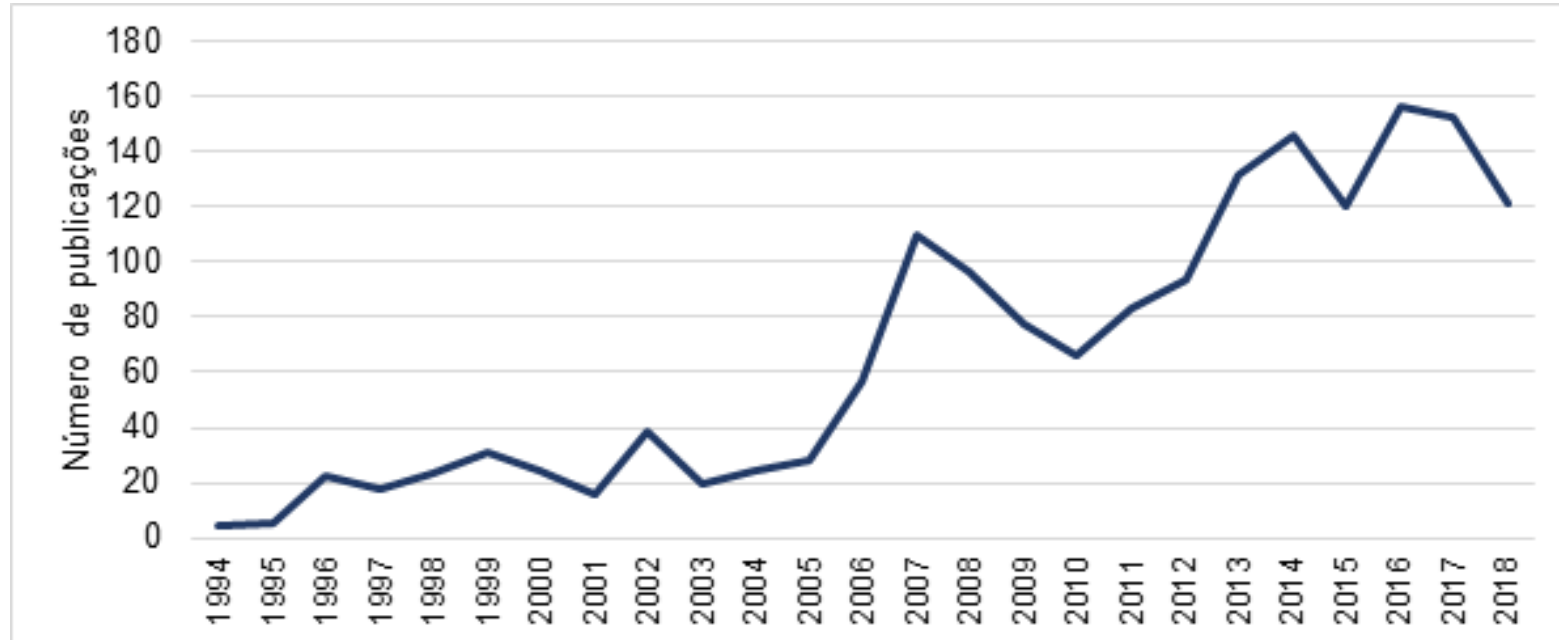

Fonte: Elaboração própria.

década de 1970, sendo apresentada a matriz insumo-produto para os anos de 1970 e 1975 (IBGE, 2008).

Os trabalhos de CUMBERLAND (1966), DALY (1969), LEONTIEF (1970), ISARD (1972) E VICTOR (1972) foram os primeiros modelos de insumo-produto que incluem interações econômicas e ambientais e, portanto, são considerados como os trabalhos seminais e balizadores para o uso desta técnica (ABDALLAH e MONTOYA, 1998). No quadro 3 é apresentado a genealogia da MIP relacionada ao meio ambiente.

CUMBERLAND (1966) é considerado como um dos primeiros a utilizar a matriz insumo-produto para questões relacionadas ao meio ambiente. Sua publicação $A$ regional inter-industry model for analysis of development objectives baseia-se no tipo setor versus setor, buscando descrever a interação entre os setores produtivos com um balanço do meio ambiente, ou seja, a diferença entre os custos e benefícios ambientais de determinado nível de produção. Segundo ABDALLAH e MONTOYA (1998), a dificuldade desse modelo consiste na correta mensuração destes dois componentes do balanço, o benefício e custo ambiental.
O modelo de DALY (1969) diferencia-se do trabalho de Cumberland em função de tratar as variáveis econômicas e ambientais em separado, criando então dois setores: humano e não humano. O lado humano é composto pelas variáveis econômicas e o lado não humano pelo meio ambiente e seus recursos naturais. Desse modo, a utilização da matriz insumo-produto de Leontief pode ser dividida em setores humanos e não humanos, aonde é possível descrever os insumos e produtos de / e para cada setor do lado humano como, por exemplo, a agricultura e, o lado não humano, dividido em setores que passam por algum processo de transformação (animais, plantas e bactérias) e setores que fornecem matéria e energia (atmosfera, hidrosfera e litosfera) (DALY, 1969).

A versão de Daly implementa uma nova forma de construir matriz insumo-produto em uma abordagem ligado ao meio ambiente, considerando produto versus setor e não setor versus setor como fez Cumberland. Contudo, o modelo de Daly possui algumas limitações, a saber: i) dificuldade em mensurar as variáveis ambientais, ou seja, como atribuir valor aos produtos ecológicos; ii) simplificação no resultado do fluxo de produção para o

Quadro 3 - Genealogia do uso da MIP para análise ligadas ao meio ambiente.

\begin{tabular}{l|l|l|l}
\hline Ano & Autor & Periódico & Título \\
\hline 1966 & John H. Cumberland & Regional Science & $\begin{array}{l}\text { A regional interindustry model for analysis of development } \\
\text { objectives }\end{array}$ \\
\hline 1969 & Herman E. Daly & $\begin{array}{l}\text { Journal of Political } \\
\text { Economy }\end{array}$ & On economics as a life science \\
\hline 1970 & Wassily Leontief & $\begin{array}{l}\text { The Review of } \\
\text { Economics and } \\
\text { Statistics }\end{array}$ & $\begin{array}{l}\text { Environmental Repercussions and the Economic Structure: } \\
\text { An Input-Output Approach }\end{array}$ \\
\hline 1972 & Weter A. Victor & New York: Free Press & $\begin{array}{l}\text { Ecologic-economic Analysis for Regional Development; } \\
\text { Some Initial Explorations with Particular Reference to } \\
\text { Recreational Resource Use and Environmental Planning }\end{array}$ \\
\hline
\end{tabular}

Fonte: Elaboração própria. 
meio ambiente, ou seja, como simplificar o fluxo de produção da atmosfera (ABDALLAH e MONTOYA, 1998).

Leontief aplica os conceitos desenvolvidos por ele sobre matriz insumo-produto, ampliando seu modelo para poder mensurar os impactos de poluição em detrimento do aumento do nível de produção. Sua base de análise é o setor versus setor. Leontief desenvolve uma matriz insumo-produto e, para as variáveis do meio ambiente, ele procura indicadores de poluição como, por exemplo, poluição do ar e, após isso, insere no seu modelo estes indicadores e demonstra o impacto ambiental a partir do fluxo de produção (ABDALLAH e MONTOYA, 1998).

Isard utilizou a abordagem insumo-produto ampliada com variáveis ecológicas e segue a estrutura de Daly, produto $\mathrm{x}$ setor. Os fluxos de insumo e produto entre os produtos e setores são descritos em coeficientes técnicos divididos em coeficientes econômicos e ambientais. A diferença principal com o modelo de Daly foi quanto à determinação dos coeficientes ambientais. Neste caso, a relação entre insumos ambientais utilizados pelo processo produtivo resulta da relação entre quantidade física ambiental do insumo e os valores da produção econômica (MIRANDA, 1980).

O modelo de VICTOR (1972) também utiliza a premissa produto versus setor e consistem em adicionar à estrutura produtiva insumos ambientais (terra, água e ar), que são demandados pelo processo produtivo, bem como, o descarte de rejeitos sobre o meio ambiente. Nes-

Figura 1 - Relação de periódicos e número de publicações

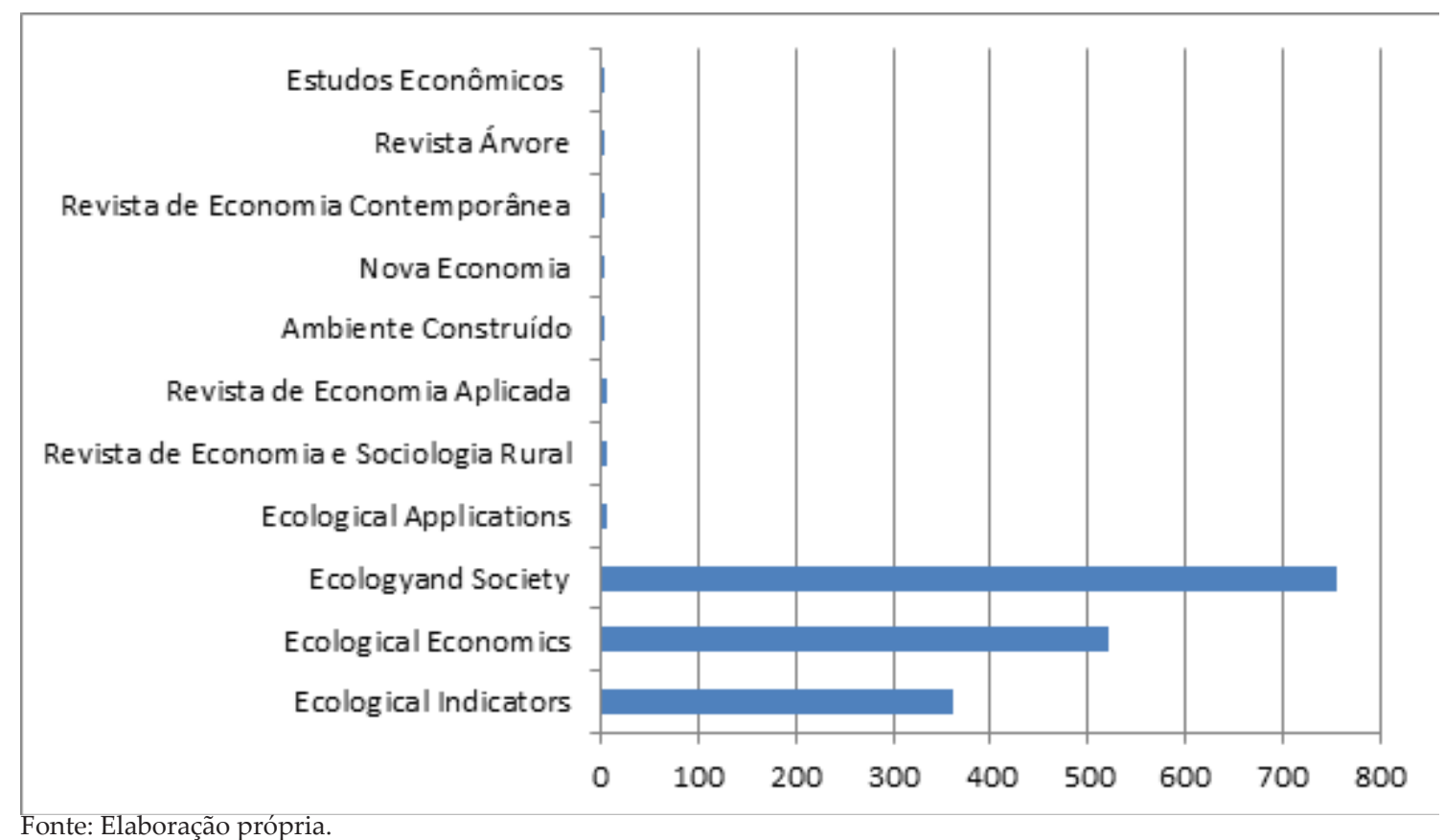

Quadro 4 - Classificação dos periódicos

\begin{tabular}{|l|l|c|}
\hline Abreviatura & Periódicos & Qualis Capes \\
\hline EI & Ecological Indicators & A1 \\
\hline EE & Ecological Economics & A1 \\
\hline ES & Ecology and Society & A1 \\
\hline EA & Ecological Applications & A1 \\
\hline SOBER & Revista de Economia e Sociologia Rural & B1 \\
\hline ECON. APL. & Revista de Economia Aplicada & B1 \\
\hline AMB. CONS. & Ambiente Construído & B1 \\
\hline NOV. ECON. & Nova Economia & B1 \\
\hline VER. ECON. CONT. & Revista de Economia Contemporânea & B1 \\
\hline ARV. & Revista Árvore & A2 \\
\hline EECON. & Estudos Econômicos & B1 \\
\hline
\end{tabular}

Fonte: Elaborador a partir de informações da QUALI-CAPES 
Quadro 5 - Artigos publicados por revista

\begin{tabular}{|c|c|c|c|c|c|c|c|c|c|c|c|}
\hline Ano & $\mathrm{EI}$ & $\mathrm{EE}$ & ES & EA & SOBER & ECON. APL. & $\begin{array}{l}\text { AMB. } \\
\text { CONS. }\end{array}$ & $\begin{array}{l}\text { NOV. } \\
\text { ECON. }\end{array}$ & $\begin{array}{l}\text { REV. } \\
\text { ECON. } \\
\text { CONT. }\end{array}$ & ARV. & EECON \\
\hline 1994 & & 4 & & & & & & & & & \\
\hline 1995 & & 5 & & & & & & & & & \\
\hline 1996 & & 5 & 17 & & & & & & & & \\
\hline 1997 & & 4 & 14 & & & & & & & & \\
\hline 1998 & & 8 & 15 & & & & & & & & \\
\hline 1999 & & 12 & 19 & & & & & & & & \\
\hline 2000 & & 12 & 12 & & & & & & & & \\
\hline 2001 & 1 & 9 & 6 & & & & & & & & \\
\hline 2002 & 3 & 13 & 23 & & & & & & & & \\
\hline 2003 & & 8 & 12 & & & & & & & & \\
\hline 2004 & 2 & 11 & 11 & & & & & & & & \\
\hline 2005 & 2 & 12 & 14 & & & & & & & & \\
\hline 2006 & 3 & 25 & 29 & & & & & & & & \\
\hline 2007 & 2 & 44 & 64 & & & & & & & & \\
\hline 2008 & 3 & 40 & 54 & & & & & & & & \\
\hline 2009 & 11 & 34 & 33 & & & & & & & & \\
\hline 2010 & 6 & 29 & 31 & & & & & & & & \\
\hline 2011 & 17 & 29 & 37 & & & & & & & & \\
\hline 2012 & 20 & 20 & 54 & & & & & & & & \\
\hline 2013 & 26 & 32 & 73 & & 1 & & & & & & \\
\hline 2014 & 40 & 33 & 69 & & 2 & & 1 & & & 1 & \\
\hline 2015 & 30 & 30 & 54 & 1 & 1 & 1 & 1 & & 1 & 1 & \\
\hline 2016 & 68 & 19 & 59 & 4 & 1 & 2 & & 1 & 1 & & 1 \\
\hline 2017 & 58 & 32 & 57 & 2 & 1 & 2 & & 1 & & & \\
\hline 2018 & 69 & 52 & & & & & & & & & \\
\hline Total & 361 & 522 & 757 & 7 & 6 & 5 & 2 & 2 & 2 & 2 & 1 \\
\hline
\end{tabular}

Fonte: Elaboração própria

se modelo, são inseridas algumas linhas referentes aos insumos ecológicos que são demandados pela produção econômica e os correspondentes subprodutos ecológicos oriundos dos processos de transformações produtivos.

Na primeira fase da pesquisa, após identificação das palavras-chaves, resultou em 1.667 artigos, distribuídos conforme apresentado na figura 1 .

$\mathrm{Na}$ figura 1, nota-se que os periódicos Ecological Applications possui 7 publicações, Revista de Economista e Sociologia Rural sete artigos, Economia aplicada 5, Ambiente Construído, Nova Economia, Economia Contemporânea e revista Árvore possuem 2 artigos e, por fim, o periódico Estudos Econômicos um.

Com base na figura 1, nota-se uma concentração significativa de publicações sobre o tema pesquisado em apenas três periódicos e número reduzido nos periódicos nacionais. Os periódicos selecionados possuem boa avaliação de impacto pelo critério da Qualis Capes conforme quadro 4.
Ressalta-se que se utilizou como critério de filtro em relação à classificação na Qualis Capes a área do conhecimento da ciência ambiental. Contudo, os periódicos Revista de Economia Aplicada, Economia Contemporânea e Estudos Econômicos não possuem classificação nesta área da ciência, no entanto, utilizou-se a classificação na área da ciência econômica para estes periódicos. O quadro 5 revela, para cada um dos periódicos selecionados, os artigos que foram publicados e o respectivo ano da publicação.

O escopo final da segunda fase da pesquisa, que foi a identificação dos trabalhos que utilizavam a matriz insumo-produto para análise do meio ambiente no Brasil, resultou em dezenove títulos cuja leitura dos mesmos se deu integralmente no período compreendido entre março e julho de 2018 e, a partir destes estudos, foi possível desenvolver uma discussão sobre matriz 
insumo-produto, evidenciando a relação entre economia e meio ambiente. Ressalta-se que esta revisão não pretendeu esgotar a pesquisa bibliográfica sobre o assunto a estes periódicos, tão pouco a aspiração de abordar todo o assunto ligado à matriz insumo-produto, mas sim coletar o número de trabalhos desenvolvidos sobre o tema a partir dos critérios supracitados procurando atender o objetivo geral da pesquisa.

Neste sentido, após a aplicação do método, os dezenove títulos selecionados no final da segunda fase da pesquisa bibliográfica, que utilizavam o meio ambiente na construção da matriz insumo-produto no Brasil, lidos integralmente nesta fase, foram analisados em ordem cronológica de publicação e notou-se pequena variação no uso dos modelos de MIP, embora com abordagens que variavam desde estadual para nacional, até para análise regional. A maior parte destas publicações concentrava-se no período entre os anos 2000 a 2016, sendo 2013 o ano com maior volume de publicação.

Em agosto de 1980, MIRANDA (1980) publicou o trabalho "Economia e Meio Ambiente: uma abordagem de insumo-produto", em que critica a carência do uso de técnicas que procuram descrever a interdependência entre setores e o meio ambiente no Brasil. Neste estudo, o autor levanta os impactos sobre a qualidade da água, em se tratando do abastecimento público, a partir das relações interindustriais da região industrial do médio rio Paraíba do Sul. O modelo utilizado foi aquele baseado em VICTOR (1972), produto versus setor, com base na regionalização da matriz insumo-produto de 1970 desenvolvida pelo IBGE. Com base neste estudo, concluiu-se que a situação do rio Paraíba, entre os anos de 1978 a 1982, não apresentava riscos para a saúde das populações em função das atividades industriais ocorridas ao longo do rio.

Outros estudos foram desenvolvidos, com a criação de matrizes regionais, como foi o caso do trabalho de SANTIAGO et al. (2010) e IMORI et al. (2014). O objetivo do trabalho de SANTIAGO et al. (2010) foi a construção de uma matriz insumo-produto híbrida para o estado de Minas Gerais, em 2005 e, a partir dela, mensurar a intensidade de emissões de $\mathrm{CO}_{2}$ em função do consumo de combustíveis na economia mineira. Neste caso, os autores, utilizando uma matriz do tipo produto versus setor do estado de Minas Gerais e, redimensionando e agregando a setores à matriz insumo-produto mineira, passando de 55 produtos e 35 setores para uma matriz agregada em 33 setores.

Levando-se em consideração o balanço energético de Minas Gerais, que contempla apenas 12 setores agregados, foi feita uma nova reorganização setorial para que a matriz insumo-produto ficasse também com 12 setores (SANTIAGO et al., 2010). A limitação desse trabalho se caracteriza pela especificidade de, quando da compatibilização dos resultados e análises, limitar-se-á apenas a alguns combustíveis e não a matriz energética como um todo.

IMORI et al. (2014) objetivou identificar a existência de possíveis compensações entre as políticas diretas de redução das emissões de gases de efeito estufa (GEEs), com as políticas de fomento ao desenvolvimento da Amazônia brasileira, considerando suas relações econômicas com o resto do país. A matriz de insumo-produto brasileira utilizada foi construída a partir de uma estimativa para 2004, de modo a permitir uma análise das relações econômicas e ambientais dos setores produtivos da região amazônica e do resto do país, tendo como modelo o tipo produto versus setor. Com o desenvolvimento desse trabalho foi possível identificar uma inversão em relação aos padrões internacionais das principais fontes de emissão de GEEs, que são causadas pela produção e energia no Brasil, e que as principais causas são mudanças de uso da terra para a agricultura.

A energia, para o caso brasileiro, não possui relativa importância porque uma parte significativa é considerada limpa. O trabalho concluiu que 30\% das emissões de $\mathrm{CO}_{2}$ na região da Amazônia brasileira são derivadas do consumo das famílias de outras regiões do Brasil e $23 \%$ das emissões de $\mathrm{CO}_{2}$ se dão em função de fatores externos como, por exemplo, a demanda por produtos (commodities) produzidos naquela região para atender outros países.

Cabe ressaltar a existência de trabalhos que procuraram descrever a relação entre a economia e o meio ambiente em escala regional para os estados de Pernambuco, Bahia, Rio Grande do Sul, São Paulo e Mato Grosso do Sul. Há ainda o registro de trabalhos desenvolvidos procurando estudar o impacto de setores e seus efeitos como, por exemplo, o trabalho de MONTOYA et al. (2013) que, a partir da matriz insumo-produto, identifica o consumo de energia, emissões de $\mathrm{CO}_{2}$ e a geração de emprego e renda no agronegócio brasileiro. Em se tratando da economia nacional, alguns estudos se destacaram e estão relacionados a seguir.

AVELINO e GUILHOTO (2009) mensuraram os impactos ambientais (emissão de CO2) envolvidos na fase de construção das usinas eólicas e o tempo de retorno para compensar essas emissões durante a operação, uma vez que a energia eólica pode ser considerada como instrumento que permita a geração de energia concomitante à redução de emissão de GEEs. A partir da matriz insumo-produto da economia brasileira para o ano de 2004 , foi possível identificar que o tempo para recuperação quando se trata dos impactos da construção das usinas de energia eólicas, é de quatro meses e, a maior contribuição de poluição deriva da produção de aço e outros produtos durante a construção.

SALVO et al. (2015) estimaram o uso e apropriação de terras no Brasil por meio da matriz insumo-produto conjugada com a análise da pegada ecológica. Foi construída, para efeitos de análise do estudo, uma matriz com 64 setores do tipo produto versus setor para o ano de 2006. Os resultados do trabalho sugerem que a melhor forma de mitigar o impacto ambiental na economia brasileira é melhorar o desempenho da agricultura e a produção de combustíveis fósseis, sendo a maior pegada ecológica a do setor da pecuária.

O estudo "O uso setorial de energia renovável versus não renovável e as emissões de CO2 na economia brasileira: um modelo insumo-produto híbrido para 53 
setores", desagrega o consumo energético brasileiro de 2009 por setor para a construção de uma matriz insumo-produto híbrida de 53 setores para avaliar as emissões de $\mathrm{CO} 2$ por fontes de energia. A pesquisa concluiu que o consumo das famílias, a partir do de aumento da renda, contribui para o aumento da demanda de energia e, por sua vez, aumento de emissão de $\mathrm{CO} 2$ no país, e que o ponto crucial para uma redução do impacto da economia sobre o meio ambiente é a mudança do comportamento do consumidor (MONTOYA e PASQUAL, 2015).

A partir do levantamento e leitura dos artigos selecionados em função de tratarem do tema matriz insumo-produto e meio ambiente diretamente, é possível identificar que os trabalhos desenvolvidos no Brasil utilizavam em sua maioria o modelo de matriz produto versus setor para análises econômica-ambiental. A hipótese central para explicar a preferência na adoção dessa técnica consiste no fato de que, os modelos setor versus setor possuem como premissa básica que um produto é produzido por um único setor exclusivamente (ABDALLAH e MONTOYA, 1998).

$\mathrm{O}$ uso de matrizes produto versus setor se sobressai em relação aos outros métodos, pois, procura rejeitar a ideia de produção de bens a partir de um único setor, ou seja, um produto pode ser produzido por mais de um setor, sendo que, esse modelo é considerado mais apropriado, pois, permitem a modelação de resíduos como subprodutos derivados da produção e consumo (FORSUND, 1985).

\section{Conclusão}

Apresenta-se, no decorrer do trabalho, os modelos iniciais de matriz insumo-produto utilizados na construção da análise econômica-ambiental. Com base na pesquisa bibliográfica em periódicos a partir das palavras-chaves matriz insumo-produto, meio ambiente e sustentabilidade foi possível encontrar 1.667 artigos com estas palavras-chaves. Desse total, desagregando em estudos que foram feitos para o Brasil, foram selecionados 19 artigos relacionados diretamente ao tema. Embora estes artigos tenham sido publicados em revistas bem avaliadas pelo Qualis Capes, esse relativo baixo número de publicações de trabalhos deste tipo se dá pelo fato da dificuldade de obtenção de dados para a construção de matrizes insumo-produto, concomitante ao fato de que o IBGE adota a construção de matrizes a cada cinco anos.

As principais características observadas nos trabalhos de análises feitas neste estudo foram: i) a maioria dos estudos utilizou o modelo produto versus setor; ii) variação no tamanho da matriz, ou seja, o número de setores estudados, modelos de matrizes variando de 12 a 64 setores, sendo que, este fato se dá em função do objetivo adotado nos respectivos estudos; iii) existências de estudos tanto no âmbito nacional quanto regional; iiii) a maioria desses estudos se baseia na construção de uma MIP híbrida energética, ou seja, a partir das variações de aumento ou diminuição de demanda de fontes de energia frente as variações de produção, quais os impactos sobre o meio ambiente em relação a produção, a partir do despejo de $\mathrm{CO}_{2}$ na atmosfera.

É possível ainda verificar que o número de trabalhos utilizando esta técnica tem aumentando ao longo dos últimos anos e, desta maneira, tem-se a oportunidade de desenvolvimento de novas pesquisas a partir do aperfeiçoamento do uso da técnica e de sua disseminação para fins de análise econômica-ambiental.

Neste sentido, ressalta-se a importância do uso da matriz insumo-produto como instrumento que permite uma análise mais profunda sobre os impactos de alterações do nível de produção e consumo de uma dada sociedade sobre o meio ambiente, procurando, desta forma, evidenciar a relação entre a economia e o meio ambiente.

\section{Referências}

ABDAllah, P. R. MONTOYA, M. A. Perspectivas da utilização de modelos insumo-produto na administração do meio ambiente. In: MONTOYA, M. A. Relações intersetoriais do Mercosul e da economia brasileira: uma abordagem de equilíbrio geral do tipo insumoproduto. Passo Fundo: EDIUPF, 1998. p. 345-365.

ARAÚJO, C. A. Bibliometria: evolução histórica e questões atuais. Em Questão. 2006; v. 12, n. 1, p. 11-32.

AVELINO, T. GUILHOTO, J. J. Ecological payback in Brazil energy matrix: analysis of a wind energy expansion. Munich Personal Repec Archive, Universidade de São Paulo, 2009.

BROWN, L. Word on the edge: how to prevent environmental and economic collapse. W.W. Norton and Company, New York and London, 2011.

CARVALHEIRO, N. Observações sobre a elaboração da matriz de insumo-produto. Pesquisa e Debate. 1998; v. 9, n. 14 , p. 139-157.

CECHIN, A. D. VEIGA, J. E. A economia ecológica e evolucionária de GeorgescuRoegen. Revista de Economia Política. 2010; v. 30, n.3, p. 438-454.

CONFORTO, E. C.; AMARAL, D. C.; SILVA, S. L. Roteiro Para Revisão Bibliográfica Sistemática: Aplicação No Desenvolvimento De Produtos E Gerenciamento De Projetos. In: 8 Congresso Brasileiro de Gestão de Desenvolvimento de Produto - CBGDP 2011, Porto Alegre. Instituto de Gestão de Desenvolvimento do Produto IGDP. Anais... Porto Alegre: IGDP, 2011. p. 1-12.

CUMBERLAND, J. H. A regional interindustry model for analysis of development objectives. Papers in Regional Science. 1966 ; ,n. 17, v. 1, p. 65-94.

CMMD. World Commission on Environment and Development: Our common future. Oxford: Oxford University Press, 1987. 
DALY, H. E. On economics as a life science. Journal of political economy. 1969; v. 76, n. 3, p 392-406.

DALY, H. E. Sustainable Development: Definitions, Principles, Policies. Invited Address, World Bank, April 30, 2002, Washington, DC.

DALY, H. E. Crescimento sustentável? Não, obrigado. Ambiente e Sociedade 2004; ,v. 7, n. 2, p. 197-201.

DENYER, D. TRANFIELD, D. Producing a systematic review. In: BUCHANAN,D. A. BRYMAN, A. The Sage handbook of organizational research methods. Thousand Oaks, CA, Sage Publications Ltd, 2009. 700p.

FEIJÓ, F. T. LAUTERT, V. BÊRNI, D. A. Contabilidade social ambiental. In: BÊRNI, D. A. LAUTERT, V. Mesoeconomia. Porto Alegre: Bookman, 2011. 661p.

FORSUND, F. R. Input-output models, national economic models and the environment. In: KNEESE, A. V., SWEENEY, J. L. Handbook of natural resource and energy economics. North-holland, Amsterdam. New York. Oxford, 1985.

GEORGESCU-ROEGEN, N. The Entropy Law and the Economic Process in Retrospect. Eastern Economic Journal, 1986; v. 12, n. 1, p. 3-25.

GUILHOTO, J. J. Input-Output Analysis: Theory and Foundations. Munich Personal Repec Archive, Universidade de São Paulo, 2011. 76p.

HARDI, P.S. B., HODGE, T. Measuring sustainable development: review of current practice. Canada: Research Publications Program, 1997.123p.

IBGE. Matriz de insumo-produto do Brasil 2000-2005. Rio de Janeiro: IBGE, 2008. 57p.

IMORI, D. GUILHOTO, J. M. DAVID, L. S. GUTIERRE, L. M. WAISMAN, C. Regional Development and Greenhouse Gases Emission: the case of the Amazon Region. In: 19th International Input-Output Conference, 06, 2014, Alexandria, Virginia. Anais... Alexandria, Virginia, 2014.

KITCHENHAM, B.A., CHARTERS, S. Guidelines for performing systematic literature reviews in software engineering. Tech. Rep., Keele University, London, 2007. 65p.

KRAUSMANN, F., GINGRICH, S., EISENMENGER, N., ERB, K.H., HABERL, H., FISCHER-KOWALSKI,M. Growth in global materials use GDP and population during the 20thcentury. Ecological Economics, London,2009; v. 68 , p. 2696-2705.
LEAL, R. A. ELY, R. A. UHR, J. G. Z. UHR, D. A. P. Ciclos econômicos e emissão de $\mathrm{CO}_{2}$ no Brasil: uma análise dinâmica para políticas ambientais ótimas. Revista Brasileira de Economia. 2015; v. 69, p. 53-73.

LEONTIEF, W. W. Quantitative Input and Output Relations in the Economic Systems of the United States. The Review of Economics and Statistics, Cambridge. 1936; v. 18, n. 3, p. 105-125.

LOUETTE, A. Indicadores de nações: uma contribuição ao diálogo da sustentabilidade. São Paulo: WHH, 2007.

MEADOWS, D. H. MEADOWS, D. L. RANDERS, J. The limits to growth. New York: Universe Books, 1972.

MILLER, R. E.; BLAIR, P. D. Input-Output Analysis: Foundations and Extensions. 2ed. Cambridge: University Press, 2009.

MIRANDA, C. R. Economia e meio ambiente: uma abordagem de insumo-produto. Pesquisa e planejamento econômico. 1980; Rio de Janeiro, v 10, n. 2, p. 601-636.

MORAN, D. D. WACKERNAGEL, M. KiZESA, J. A. GOLDFINGERA, S. H. AURÉLIEN, B. Measuring sustainable development: Nation by nation. Ecological economics, 2008; v 64, p. 470-474.

MONTOYA, M. A. PASQUAL, C. A. LOPES, R. L. GUILhoto, J. M. Consumo de Energia, Emissões de CO2 e a Geração de Emprego e Renda no Agronegócio Brasileiro: Uma Análise Insumo-Produto. Economia aplicada. 2013; v. 20, n. 4, p. 338-412.

MONTOYA, M. A. PASQUAL, C. A. O uso setorial de energia renovável versus não renovável e as emissões de co2 na economia brasileira: um modelo insumoproduto híbrido para 53 setores. Pesquisa e planejamento econômico. 2015; v. 45, n. 2, p. 290-335.

RICHARDSON, H. W. Insumo-produto e economia regional. Rio de Janeiro: Zahar, 1978.

ROSSETTI, J. P. Contabilidade social: uma abordagem introdutória. São Paulo: Atlas, 1995.

ROYAL SOCIETY. People and the Planet. The Royal Society, London, 2012.

SANTIAGO, T. S. CARVALHO, F. S. PEROBELLI, F. Análise setorial da intensidade de emissões de co2 e na estrutura de exportações: um modelo regional de insumoproduto para minas gerais. In: XIV Seminário sobre a Economia Mineira, 2010, Minas Gerais. Anais... Minas Gerais: UFMG, 2010,p. 1-18. 
SALVO, G. SIMAS, M. S. PACCA, S. A. GUILHOTO, J. M. TOMAS, A. R. G. ABRAMOVAY, R. Estimating the human appropriation of land in Brazil by means of an Input-Output Economic Model and Ecological Footprint analysis. Ecological Indicators. 2015; v. 53, p. 78-94.

SUlSTON, J. RUMSBY, M. GREEN, N. People and the Planet. Environment Resource Economy. 2013; v. 55, n. 4 , p. $469-474,2013$.

SCHMITZ, A. P. BITTENCOURT, M. V. L. Crescimento econômico e pressão sobre recursos hídricos. Estudos Econômicos, São Paulo, v. 47, n. 2, p. 329-363, 2017.

UNEP. Global Material flows and resource productivity. New York: UN Sales Publication, 2016.

VICTOR, P. A. Pollution economic and environment. London, George Allen \& Unwin, 1972.

\section{Contribuição dos autores}

Raul Asseff Castelao

Contribuições substanciais para a concepção do trabalho; análise e interpretação de dados.

Celso Correia de Souza

Contribuições substanciais para a concepção do trabalho; análise e

interpretação de dados; redigiu o trabalho ou realizou uma revisão substancial.

Daniel Massen Frainer

Contribuições substanciais para a concepção do trabalho; análise e

interpretação de dados; redigiu o trabalho ou realizou uma revisão substancial. 